\title{
MTORC1/mTORC2/DHFR Inhibitor ABTL0812
}

National Cancer Institute

\section{Source}

National Cancer Institute. mTORC1/mTORC2IDHFR Inhibitor ABTL0812. NCI Thesaurus.

Code C117290.

An orally bioavailable, lipid analogue and inhibitor of raptor-mammalian targ et of rapamycin (mTOR) (mTOR complex 1; mTORC1), rictor-mTOR (mTOR complex 2;

mTORC2) and dihydrofolate reductase (DHFR) with potential antineoplastic activity. Upon oral administration, mT ORC1/mT ORC2/DHFR inhibitor ABT L0812 binds to and inhibits both mTORC1 and mTORC2, which may result in apoptosis and a decrease in proliferation in mTORC1/2-expressing tumor cells. mTOR is a serine/threonine kinase that is upregulated in some tumors; it plays an important role in the PI3K/Akt/mTOR signaling pathway which is often deregulated in cancer cells. In addition, ABT L0812 inhibits DHFR, an enzyme that reduces dihydrofolic acid to tetrahydrofolic acid, thereby blocking tetrahydrofolate synthesis, and resulting in both the depletion of nucleotide precursors and the inhibition of DNA, RNA and protein synthesis. This induces autophagy-induced cell death and further inhibition of cell proliferation. 